\title{
Clustering with Multiple Receiving Antennas in Downlink FDD CoMP Systems
}

\author{
P. Baracca ${ }^{\dagger}$, F. Boccardi ${ }^{\dagger}$, and N. Benvenuto* \\ $\dagger$ Bell Laboratories, Alcatel-Lucent. Email: \{paolo.baracca, federico.boccardi\}@alcatel-lucent.com \\ * Department of Information Engineering, University of Padova, Italy. Email: nb@dei.unipd.it
}

\begin{abstract}
Inter-cell interference in downlink cellular networks can be managed by coordination among the base stations (BSs). Constraints on the backhaul throughput make full coordination still challenging and typically clusters of BSs are organized to serve the user equipments (UEs). Joint precoding within each cluster is designed to deal with intra-cluster interference. Moreover, inter-cluster interference (ICI) can be reduced by implementing dynamic clustering, i.e., by changing BS clusters over time to provide more fairness among the UEs. In this work we assume that UEs are equipped with multiple antennas and use an interference rejection combiner to suppress the ICI not managed by precoders at transmitter side. In this framework, we develop an algorithm that dynamically organizes clusters and schedules UEs in each cluster by requiring a channel state information at the transmitter which is independent of the number of receiving antennas. Simulations provide two main results: a) a considerable improvement is achieved by adding antennas at the UE and $b$ ) the gain of dynamic clustering over static clustering sensibly decreases by equipping the UEs with more antennas.
\end{abstract}

\section{INTRODUCTION}

In cellular downlink networks coordinated multi-point (CoMP) schemes provide a huge gain with respect to single cell processing (SCP) schemes: the performance degradation in SCP due to inter-cell interference can be perfectly nulled by allowing full sharing of user equipment (UE) data and channel state information (CSI) among the base stations (BSs), which, in turn, serve the scheduled UEs by using joint precoding [1]. This scheme is also known in literature as CoMP with joint processing (JP), opposed to CoMP with coordinated beamforming $(\mathrm{CB})$ where only CSI is shared among the BSs and inter-cell interference is reduced by properly designing the precoder used by each BS while serving its own UEs [2]. Even if CoMP-JP is a very promising technique, many practical constraints make its implementation still challenging. First of all, a backhaul network able to support UE data sharing among the BSs is required. Typically, clusters of BSs are organized and joint precoding is implemented only within each cluster. However, UEs at the cluster border still suffer intercluster interference (ICI), which is managed either by dynamic clustering [3], where clusters change over time adapting to channel conditions, or by designing precoders in each cluster in order to limit the ICI caused to UEs in neighbouring clusters [4]. A second issue is that CSI at the BSs may be unreliable because of noise on channel estimation in time division duplex (TDD) systems or limited bandwidth available for feedback in frequency division duplex (FDD) systems.

Most of the works on dynamic clustering [3], [5], [6], [7], assume that UEs are equipped with only one antenna, and propose different methods to optimize clusters [3], [5], also jointly either with feedback design [6] or UE selection [7]. However, the LTE-Advanced standard developed by the 3rd Generation Partnership Project (3GPP) assumes that UEs may be equipped with up to eight antennas [8]. Even if this number seems a bit optimistic for current mobile devices, the technological innovation may allow in the near-future manufacturing smartphones or tablets with numerous antennas. Thus, much more attention should be paid to the study of CoMP schemes when UEs may exploit the benefits of multiple antennas [9].

In this work we study the achievable performance in downlink FDD CoMP systems when UEs are equipped with multiple antennas. We start from the dynamic clustering and scheduling algorithm proposed in [10]. To simplify CSI at the transmitter side, we consider a feedback scheme that requires a feedback bandwidth independent of the number of UE antennas. Moreover, we also assume that BSs transmit only one stream of data to the scheduled UEs, which, in turn, implement an interference rejection combiner (IRC) [11] to suppress the residual interference. Then, we compare the proposed strategy against both static clustering, where clusters do not change over time, and single cell processing, where no cooperation among the BSs is allowed. Numerical results show that a) an important gain is achieved by equipping UEs with many antennas to suppress ICI and b) the gain of dynamic clustering over static clustering and SCP sensibly decreases as the number of UE antennas increases. Hence, the main finding of this paper is that the complex operation of dynamic clustering is not worthy when the number of UE antennas is large enough. Notation: We use $(\cdot)^{T}$ to denote transpose and $(\cdot)^{H}$ conjugate transpose. $\mathbf{0}_{N \times M}$ denotes the matrix of size $N \times M$ with all zero entries, $\boldsymbol{I}_{N}$ the identity matrix of size $N, \operatorname{tr}(\boldsymbol{X})$ the trace of matrix $\boldsymbol{X},[\boldsymbol{X}]_{n, m}$ the entry on row $n$ and column $m$ of $\boldsymbol{X}$, and $[\boldsymbol{X}]_{,, m}$ the $m$-th column of $\boldsymbol{X}$.

\section{SySTEM MODEL}

We consider a system where a set $\mathcal{J}=\{1,2, \ldots, J\}$ of BSs, each equipped with $M$ antennas, is serving a set $\mathcal{K}=\{1,2, \ldots, K\}$ of UEs, each equipped with $N$ antennas, with $K \geq J M$. As the overall number of transmitting antennas 
is not sufficient to serve all the UEs in each time slot, UE scheduling is part of the optimization problem. We assume flat-fading channels and denote with $\boldsymbol{H}_{k, j}(t)$ the multipleinput multiple-output (MIMO) channel matrix of size $N \times M$ between BS $j$ and UE $k$ in time slot $t$. We assume that entries of matrix $\boldsymbol{H}_{k, j}(t)$ are identically distributed zero-mean complex Gaussian random variables, i.e., $\left[\boldsymbol{H}_{k, j}(t)\right]_{n, m} \sim$ $\mathcal{C N}\left(0, \sigma_{k, j}^{2}\right), n=0,1, \ldots, N-1, m=0,1, \ldots, M-1$. Note that $\sigma_{k, j}^{2}$ represents the large scale fading between UE $k$ and $\mathrm{BS} j$, which depends on path-loss and shadowing. We also assume that channels are correlated by considering the popular Kronecker model [12]. By denoting with $\boldsymbol{R}_{\mathrm{BS}}$ the square correlation matrix of size $M$ at the BS, with $\operatorname{tr}\left(\boldsymbol{R}_{\mathrm{BS}}\right)=M$, and with $\boldsymbol{R}_{\mathrm{UE}}$ the square correlation matrix of size $N$ at the UE, with $\operatorname{tr}\left(\boldsymbol{R}_{\mathrm{UE}}\right)=N$, we can write

$$
\boldsymbol{H}_{k, j}(t)=\boldsymbol{R}_{\mathrm{UE}}^{1 / 2} \overline{\boldsymbol{H}}_{k, j}(t)\left(\boldsymbol{R}_{\mathrm{BS}}^{1 / 2}\right)^{H}
$$

where $\overline{\boldsymbol{H}}_{k, j}(t)$ is a matrix of size $N \times M$ whose entries are independent and identically distributed zero-mean complex Gaussian random variables with $\sigma_{k, j}^{2}$ as statistical power. More on $\boldsymbol{R}_{\mathrm{BS}}$ and $\boldsymbol{R}_{\mathrm{UE}}$ in Section V.

We denote with $j_{k}$ the anchor BS of UE $k$, which is defined as the BS characterized by the highest signal to noise ratio (SNR) averaged with respect to fading, i.e.,

$$
j_{k}=\underset{j \in \mathcal{J}}{\operatorname{argmax}} \sigma_{k, j}^{2} \text {. }
$$

We assume that a central unit $(\mathrm{CU})$ coordinates the BSs by a) organizing in each time slot a set of clusters and b) scheduling a set of UEs within each cluster.

\section{CSI RECOVERY AT THE CU}

In the downlink, obtaining CSI at the BSs for precoding design is in general a bigger issue than obtaining CSI at the UE for reception: in fact, in FDD there is only a limited bandwidth available for feedback. Hence, in the rest of the paper we assume that the $\mathrm{CU}$ does not know all the channels, whereas perfect CSI is available at the UE. Similarly to [6], UE $k$ selects a subset of preferred BSs $\mathcal{J}_{k} \subseteq \mathcal{J}$, and reports only a feedback on the channels connecting itself to the BSs in $\mathcal{J}_{k}$. We consider that $\mathcal{J}_{k}$ includes only the BSs whose long term SNR is within a certain range $\epsilon_{\mathrm{TH}}$ with respect to the long term SNR between UE $k$ and its anchor BS $j_{k}$, i.e.,

$$
\mathcal{J}_{k}=\left\{j_{k}\right\} \cup\left\{j \in \mathcal{J}: \sigma_{k, j}^{2} \geq \epsilon_{\mathrm{TH}} \sigma_{k, j_{k}}^{2}\right\} .
$$

Moreover, we also consider a maximum size for set $\mathcal{J}_{k}$ by imposing $\left|\mathcal{J}_{k}\right| \leq J_{\text {MAX }}, k \in \mathcal{K}$. Note that in (3) a wider CSI is available at the $\mathrm{CU}$ when lower values of $\epsilon_{\mathrm{TH}}$ are used. However, in a practical system, the size of $\mathcal{J}_{k}$, and hence the value of $\epsilon_{\mathrm{TH}}$, depends on the number $M$ of transmitting antennas, the number $K$ of UEs in the network and the coherence time of the channel. Indeed, due to the limited bandwidth available for feedback, more bits are required to reliably quantize the channel when $M$ increases, fewer bits are available to each UE when the number $K$ of active UEs increases, and the feedback needs to be sent more often when the channel changes rapidly.

Multiple antennas at the receiver could be used by the UE to detect multiple streams of data. However, here we assume rank-1 transmission, i.e., each cluster of BSs sends only one stream of data to each UE scheduled within the cluster. Hence, after defining the bijective function $\Psi_{k}:\left\{1,2, \ldots,\left|\mathcal{J}_{k}\right|\right\} \rightarrow$ $\mathcal{J}$ which maps BSs in $\mathcal{J}_{k}$ to set $\mathcal{J}$, we denote the MIMO channel matrix of size $N \times\left|\mathcal{J}_{k}\right| M$ connecting BSs in $\mathcal{J}_{k}$ to UE $k$ with $\hat{\boldsymbol{H}}_{k}=\left[\boldsymbol{H}_{k, \Psi_{k}(1)}, \boldsymbol{H}_{k, \Psi_{k}(2)}, \ldots, \boldsymbol{H}_{k, \Psi_{k}\left(\left|\mathcal{J}_{k}\right|\right)}\right]$. After computing its singular value decomposition (SVD), we can write $\hat{\boldsymbol{H}}_{k}=\hat{\boldsymbol{U}}_{k} \hat{\boldsymbol{\Sigma}}_{k} \hat{\boldsymbol{V}}_{k}^{H}$, where the singular values in $\hat{\boldsymbol{\Sigma}}_{k}$ are in decreasing order, i.e., $\left[\hat{\boldsymbol{\Sigma}}_{k}\right]_{s_{1}, s_{1}} \geq\left[\hat{\boldsymbol{\Sigma}}_{k}\right]_{s_{2}, s_{2}}$ when $s_{1}<$ $s_{2}$, and UE $k$ feeds back an equivalent multiple-input singleoutput (MISO) channel $\hat{\boldsymbol{h}}_{k}$ of size $1 \times\left|\mathcal{J}_{k}\right| M$ connecting BSs in $\mathcal{J}_{k}$ to itself. We consider that $\hat{\boldsymbol{h}}_{k}$ is a scaled version of the right singular vector related to the largest singular value, i.e.,

$$
\begin{aligned}
\hat{\boldsymbol{h}}_{k} & =\left[\hat{\boldsymbol{h}}_{k, \Psi_{k}(1)}, \hat{\boldsymbol{h}}_{k, \Psi_{k}(2)}, \ldots, \hat{\boldsymbol{h}}_{\left.k, \Psi_{k}\left(\left|\mathcal{J}_{k}\right|\right)\right]}\right. \\
& =\left[\hat{\boldsymbol{\Sigma}}_{k}\right]_{0,0}\left[\hat{\boldsymbol{V}}_{k}\right]_{,, 0}^{H}
\end{aligned}
$$

Note that in (4) $\hat{\boldsymbol{h}}_{k, j}, j \in \mathcal{J}_{k}$, denotes the equivalent MISO channel between BS $j \in \mathcal{J}_{k}$ and UE $k$ recovered at the CU. Under this assumption, as the CU does not know the channels connecting $\mathrm{BSs}$ outside $\mathcal{J}_{k}$ to $\mathrm{UE} k$, in the following we consider

$$
\hat{\boldsymbol{h}}_{k, j}=\mathbf{0}_{1 \times M}, \quad j \notin \mathcal{J}_{k} .
$$

We underline that in a FDD system, the bandwidth required for the feedback of $\hat{\boldsymbol{h}}_{k}$ in (4) is independent of the number $N$ of receiving antennas.

\section{Dynamic Clustering and Scheduling}

In this section we extend the algorithm proposed in [10] for dynamic clustering and scheduling to the considered scenario, where now each UE is equipped with multiple antennas.

As the number of possible clusters increases exponentially with $J$, to limit the complexity of the clustering problem we assume that the $\mathrm{CU}$ defines, on the basis of the large scale fading, a set of candidate BS clusters $\mathcal{C}=\left\{\mathcal{C}_{1}, \mathcal{C}_{2}, \ldots, \mathcal{C}_{C}\right\}$, with $\mathcal{C}_{c} \subseteq \mathcal{J}$. Note that $\mathcal{C}$ needs to be updated only when the channel statistical power changes. Here we select $C \leq K$ while $\mathcal{C}$ includes all and only the sets of preferred BSs $\mathcal{J}_{k}$ indicated by UE $k(3)$, i.e.,

$$
\forall c \in\{1,2, \ldots, C\}, \exists k \in \mathcal{K}: \mathcal{C}_{c}=\mathcal{J}_{k} .
$$

We denote with $\mathcal{U}_{c}$ the set of UEs that can be scheduled in candidate cluster $c$, which includes all the UEs whose anchor BS belongs to $\mathcal{C}_{c}$, i.e.,

$$
\mathcal{U}_{c}=\left\{k \in \mathcal{K}: j_{k} \in \mathcal{C}_{c}\right\} .
$$

Then, in each time slot $t$, the CU organizes BS clusters and schedules UEs in each cluster by applying the following two-step algorithm. 
1) For each candidate BS cluster $c$, the CU estimates a weighted sum rate $\hat{R}_{c}(t)$ by selecting a set of UEs $\mathcal{S}_{c}(t) \subseteq \mathcal{U}_{c}$, designing precoders and allocating powers.

2) With the aim of maximizing the system weighted sum rate, the $\mathrm{CU}$ schedules a set of non-overlapping $\mathrm{BS}$ clusters where each BS belongs to at most one cluster. In the rest of this section we describe more in detail these two main steps of the algorithm by dropping time index $t$ for the sake of clarity.

\section{A. Cluster Weighted Sum Rate Estimation}

By defining the bijective function $\Upsilon_{c}:\left\{1,2, \ldots,\left|\mathcal{C}_{c}\right|\right\} \rightarrow \mathcal{J}$ which maps BSs in cluster $c$ to set $\mathcal{J}$, we denote with a) $\hat{\boldsymbol{h}}_{k}^{(c)}=\left[\hat{\boldsymbol{h}}_{k, \Upsilon_{c}(1)}, \hat{\boldsymbol{h}}_{k, \Upsilon_{c}(2)}, \ldots, \hat{\boldsymbol{h}}_{k, \Upsilon_{c}\left(\left|\mathcal{C}_{c}\right|\right)}\right]$ the row vector collecting the equivalent MISO channel after receive beamforming between BS cluster $c$ and UE $k$, b) $\boldsymbol{g}_{k}^{(c)}=$ $\left[\boldsymbol{g}_{k, \Upsilon_{c}(1)}^{T}, \boldsymbol{g}_{k, \Upsilon_{c}(2)}^{T}, \ldots, \boldsymbol{g}_{k, \Upsilon_{c}\left(\left|\mathcal{C}_{c}\right|\right)}^{T}\right]^{T}$ the unitary-norm precoder used by BS cluster $c$ to serve UE $k$ and c) $P_{k}$ the corresponding allocated power. By considering a maximum power $\bar{P}$ available at each BS, the CU estimates a weighted sum rate $\hat{R}_{c}$ for cluster $c$ by solving the following optimization problem

$$
\begin{aligned}
& \hat{R}_{c}=\max _{P_{k}, \boldsymbol{g}_{k}^{(c)}, \mathcal{S}_{c} \subseteq \mathcal{U}_{c}} \sum_{k \in \mathcal{S}_{c}} \alpha_{k} \times \\
& \log _{2}\left(1+\frac{\left|\hat{\boldsymbol{h}}_{k}^{(c)} \boldsymbol{g}_{k}^{(c)}\right|^{2} P_{k}}{\sigma_{n}^{2}+\xi_{k}^{(c)}+\sum_{m \in \mathcal{S}_{c}, m \neq k}\left|\hat{\boldsymbol{h}}_{k}^{(c)} \boldsymbol{g}_{m}^{(c)}\right|^{2} P_{m}}\right),
\end{aligned}
$$

s.t.

$$
\sum_{k \in \mathcal{S}_{c}}\left\|\boldsymbol{g}_{k, j}\right\|^{2} P_{k} \leq \bar{P}, \quad j \in \mathcal{C}_{c},
$$

where $\alpha_{k}$ is the quality of service (QoS) for $\mathrm{UE} k, \sigma_{n}^{2}$ is the thermal noise power and $\xi_{k}^{(c)}$ is an estimate of the residual interference suffered by UE $k$ including ICI and interference due to the limited CSI available at the CU. With the aim of solving (8) independently of other clusters, we use the approximation

$$
\xi_{k}^{(c)}=\frac{\bar{P}}{M} \sum_{j \in \mathcal{J}_{k} \backslash \mathcal{C}_{c}}\left\|\hat{\boldsymbol{h}}_{k, j}\right\|^{2}+\bar{P} \sum_{j \in \mathcal{J} \backslash \mathcal{J}_{k}} \sigma_{k, j}^{2} .
$$

In (9) the first summation approximates the ICI due to the preferred BSs outside cluster $c$, whereas the second summation approximates the interference due to the BSs not included in set $\mathcal{J}_{k}$, i.e., whose channels are not known at the CU.

To solve problem (8) we need to specify the scheduling algorithm and the type of precoder. In Section $\mathrm{V}$ we solve (8) by considering a) zero forcing beamforming (to null the intra-cluster interference), b) equal power allocation among the scheduled UEs, and c) a greedy iterative UE selection algorithm which, at each iteration, selects the best UE in $\mathcal{U}_{c}$ not yet included in $\mathcal{S}_{c}$ by maximizing the objective function (8a).

\section{B. Clustering Optimization}

After evaluating the weighted sum rate (8) for each candidate cluster, the $\mathrm{CU}$ schedules a set of non-overlapping clusters by maximizing the system weighted sum rate. In detail, by defining

$$
\begin{aligned}
a_{j, c} & = \begin{cases}1, & j \in \mathcal{C}_{c} \\
0, & \text { otherwise }\end{cases} \\
x_{c} & = \begin{cases}1, & \mathrm{CU} \text { schedules cluster } c \\
0, & \text { otherwise }\end{cases}
\end{aligned}
$$

we consider that each BS belongs to at most one cluster by imposing

$$
\sum_{c=1}^{C} a_{j, c} x_{c} \leq 1, \quad j \in \mathcal{J} .
$$

Then, at the CU the cluster selection is performed by solving the following integer optimization problem

$$
\begin{gathered}
\max _{x_{c} \in\{0,1\}} \sum_{c=1}^{C} \hat{R}_{c} x_{c}, \\
\text { s.t. }(10) .
\end{gathered}
$$

As (11) turns out to be an NP-complete problem, we decide to solve it by using a greedy iterative algorithm which, at each iteration, selects the cluster that maximizes the per-BS weighted sum rate $\hat{R}_{c} /\left|\mathcal{C}_{c}\right|$ and satisfies constraint (10).

By denoting with $x_{c}^{(*)}, c=1,2, \ldots, C$, the greedy solution to (11), the set $\mathcal{S}$ of UEs scheduled in time slot $t$ turns out to be

$$
\mathcal{S}=\bigcup_{c: x_{c}^{(*)}=1} \mathcal{S}_{c}
$$

Moreover, BSs in cluster $c$, with $x_{c}^{(*)}=1$, serve UE $k \in \mathcal{S}_{c}$ by employing beamformer $\boldsymbol{g}_{k}^{(c)}$.

\section{Rate Computation}

By defining vector $\boldsymbol{g}_{k}=\left[\boldsymbol{g}_{k, 1}^{T}, \boldsymbol{g}_{k, 2}^{T}, \ldots, \boldsymbol{g}_{k, J}^{T}\right]^{T}$, and matrix $\boldsymbol{H}_{k}=\left[\boldsymbol{H}_{k, 1}, \boldsymbol{H}_{k, 2}, \ldots, \boldsymbol{H}_{k, J}\right]$, the signal received by UE $k$ in time slot $t$ can be written as

$$
\boldsymbol{r}_{k}=\boldsymbol{H}_{k} \boldsymbol{g}_{k} s_{k}+\sum_{m \in \mathcal{S} \backslash\{k\}} \boldsymbol{H}_{k} \boldsymbol{g}_{m} s_{m}+\boldsymbol{n},
$$

where $s_{k} \sim \mathcal{C N}\left(0, P_{k}\right)$ is the data symbol transmitted toward UE $k$ and $\boldsymbol{n} \sim \mathcal{C N}\left(\mathbf{0}_{N \times 1}, \sigma_{n}^{2} \boldsymbol{I}_{N}\right)$ is the thermal noise at the UE antennas. A linear combiner $\boldsymbol{w}_{k}$ of size $1 \times N$ is applied by UE $k$ to $\boldsymbol{r}_{k}$ thus yielding $y_{k}=\boldsymbol{w}_{k} \boldsymbol{r}_{k}$, which is used for the detection of $s_{k}$. As we are assuming perfect CSI at the receiver, UEs employ IRC, which both minimizes the mean square error and maximizes the signal to interference plus noise ratio (SINR) at the detection point [11], i.e.,

$\boldsymbol{w}_{k}=P_{k} \boldsymbol{g}_{k}^{H} \boldsymbol{H}_{k}^{H}\left(\sigma_{n}^{2} \boldsymbol{I}_{N}+\sum_{m \in \mathcal{S} \backslash\{k\}} P_{m} \boldsymbol{H}_{k} \boldsymbol{g}_{m} \boldsymbol{g}_{m}^{H} \boldsymbol{H}_{k}^{H}\right)^{-1}$. 


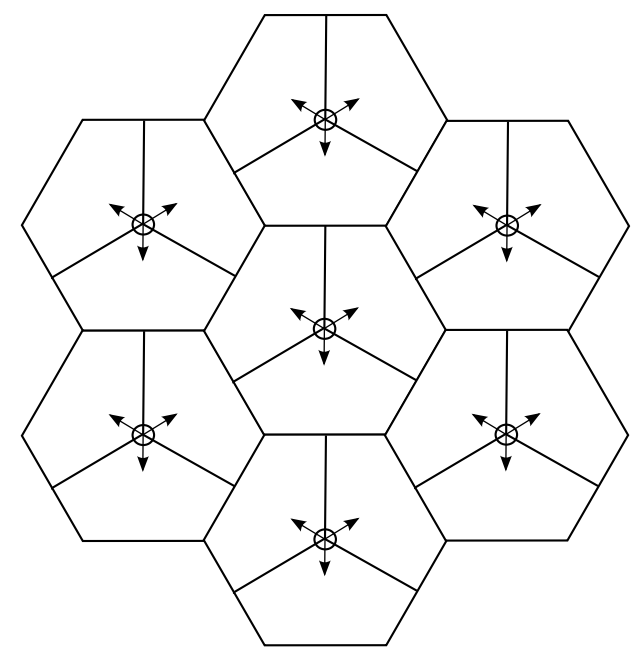

Fig. 1. Simulation setup with $J=21 \mathrm{BSs}$ organized in 7 sites, each with 3 sectors.

To implement IRC (14), UE $k$ needs to know both the equivalent channel $\boldsymbol{H}_{k} \boldsymbol{g}_{k}$, which can be estimated thanks to a training sequence sent by its assigned BS cluster, and the interfering signals $\boldsymbol{H}_{k} \boldsymbol{g}_{m}, m \in \mathcal{S} \backslash\{k\}$, which can be still estimated at the UE $k$ by exploiting the training sequences sent by the interfering BS clusters toward the co-scheduled UEs.

Finally, from the expression of $y_{k}$, the spectral efficiency achieved by UE $k \in \mathcal{S}$ can be written as

$$
R_{k}=\log _{2}\left(1+\frac{\left|\boldsymbol{w}_{k} \boldsymbol{H}_{k} \boldsymbol{g}_{k}\right|^{2} P_{k}}{\sigma_{n}^{2}\left\|\boldsymbol{w}_{k}\right\|^{2}+\sum_{m \in \mathcal{S} \backslash\{k\}}\left|\boldsymbol{w}_{k} \boldsymbol{H}_{k} \boldsymbol{g}_{m}\right|^{2} P_{m}}\right) .
$$

\section{Numerical Results}

We consider an hexagonal cellular scenario where $J=21$ BSs, each equipped with $M=4$ antennas, are grouped in 7 sites, each with 3 co-located BSs (see also Fig. 1). We consider 10 UEs randomly dropped in the coverage area of each BS, with $K=210$ UEs overall. The power available at each BS is $\bar{P}=46 \mathrm{dBm}$ and the thermal noise power at the UE is $\sigma_{n}^{2}=-101 \mathrm{dBm}$. The large scale fading between UE $k$ and BS $j$ can be written as

$$
\sigma_{k, j}^{2}=\Gamma^{(\mathrm{CE})}\left(\frac{d^{(\mathrm{CE})}}{d_{k, j}}\right)^{\eta} e^{\zeta_{k, j}} A\left(\theta_{k, j}\right),
$$

where $d_{k, j}$ is the distance between $\mathrm{UE} k$ and $\mathrm{BS} j, \eta=3.5$ is the path-loss coefficient, $\left.\Gamma^{(\mathrm{CE})}\right|_{\mathrm{dB}}=10 \mathrm{~dB}$ is the long-term SNR when a UE is at the cell edge, $e^{\zeta_{k, j}}$ is the lognormal shadowing with $8 \mathrm{~dB}$ as standard deviation and $A\left(\theta_{k, j}\right)$ models the antenna gain as a function of the direction $\theta_{k, j}$ of UE $k$ with respect to the antennas of BS $j$, with $\left.A\left(\theta_{k, j}\right)\right|_{\mathrm{dB}}=$ $-\min \left\{12\left(\theta_{k, j} / \theta_{3 \mathrm{~dB}}\right)^{2}, A_{s}\right\}$, where $\theta_{3 d B}=(70 / 180) \pi$ and $\left.A_{s}\right|_{\mathrm{dB}}=20 \mathrm{~dB}[13,(21.3)]$. We consider an inter-site distance

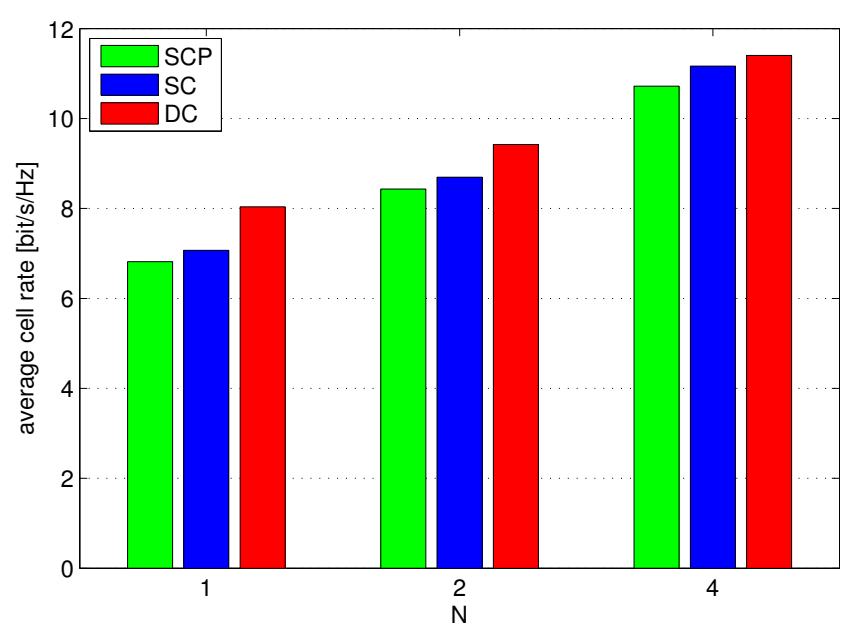

Fig. 2. Average cell rate in terms of $N$ when $M=4$.

of $500 \mathrm{~m}$ and a minimum distance $d_{\min }=35 \mathrm{~m}$ between BSs and UEs. Wraparound is used to deal with boundary effects.

Results are obtained by simulating 100 UE drops and $T=200$ channel realizations for each drop. We assume that proportional fair scheduling is implemented to provide fairness among the UEs, i.e., $\alpha_{k}(t)=1 / \bar{R}_{k}(t)$, with $\bar{R}_{k}(t+$ $1)=(1-\gamma) \bar{R}_{k}(t)+\gamma R_{k}(t), t=1,2, \ldots, T$, where $\gamma=0.1$ is the forgetting factor and we initialize $\bar{R}_{k}(1)=$ $\log _{2}\left(1+\bar{P} \sigma_{k, j_{k}}^{2} / \sigma_{n}^{2}\right)$. However, to allow the scheduler to reach a steady state, only the last $T / 2$ channels are considered for system performance evaluation.

We compare the developed scheme based on dynamic clustering (DC) with $J_{\mathrm{MAX}}=4$, against a) SCP, where each $\mathrm{UE}$ is served always by its anchor BS and no cooperation is allowed among the BSs, and b) static clustering (SC), where each UE is served by the $3 \mathrm{BSs}$ of the closest site, i.e., in the simulation setup of Fig. 1, we consider 7 clusters, each one composed by 3 co-located BSs. In order to fairly compare DC and SC in terms of CSI at the CU, and therefore in terms of bandwidth required for feedback, we numerically set $\epsilon_{\mathrm{TH}}=-14.51 \mathrm{~dB}$ in (3), such that $\mathbb{E}\left[\left|\mathcal{J}_{k}\right|\right]=3$ when DC is considered. As introduced in Section III, we underline that the $\mathrm{CSI}$ at the CU for all the considered schemes is independent of the number $N$ of receiving antennas.

First, we assume uncorrelated antennas, i.e., in (1) $\boldsymbol{R}_{\mathrm{BS}}=$ $\boldsymbol{I}_{M}$ and $\boldsymbol{R}_{\mathrm{UE}}=\boldsymbol{I}_{N}$, and evaluate the impact of the number $N$ of receiving antennas by reporting the average cell rate and the 5th percentile of the UE rate in Fig.s 2 and 3, respectively. By adding antennas at the UE, we observe an important performance improvement as IRC is able to strongly limit the impact of the residual ICI not managed at the transmitter side. For instance, by increasing $N$ from 1 to 4 , there is a gain of about $80 \%$ in terms of 5 th percentile of the UE rate with SCP. Then, we observe that SC provides a moderate gain with respect to SCP in terms of cell rate, but almost no gain in terms of the 5th percentile of the UE rate. Indeed SC, by simply allowing cooperation among the sectors of the same 


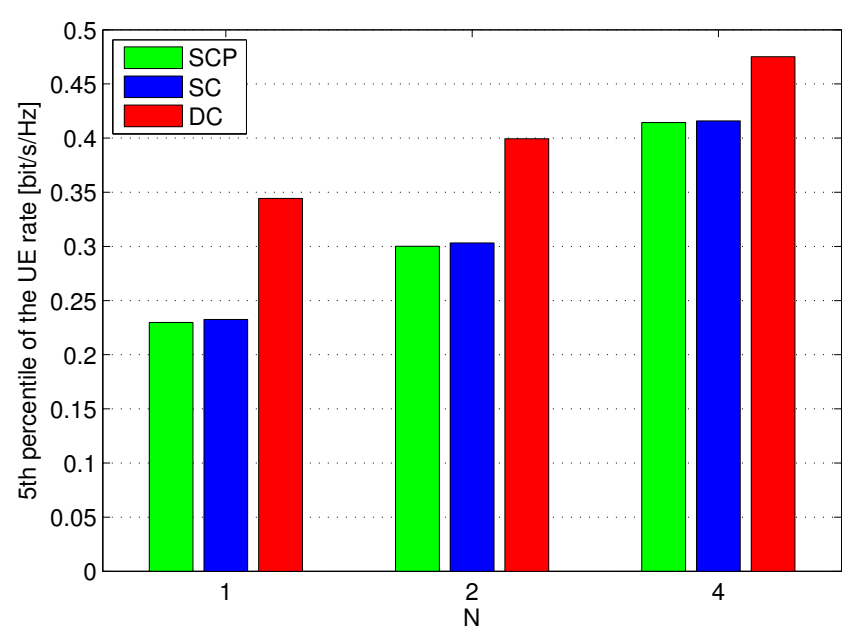

Fig. 3. 5th percentile of the UE rate in terms of $N$ when $M=4$.

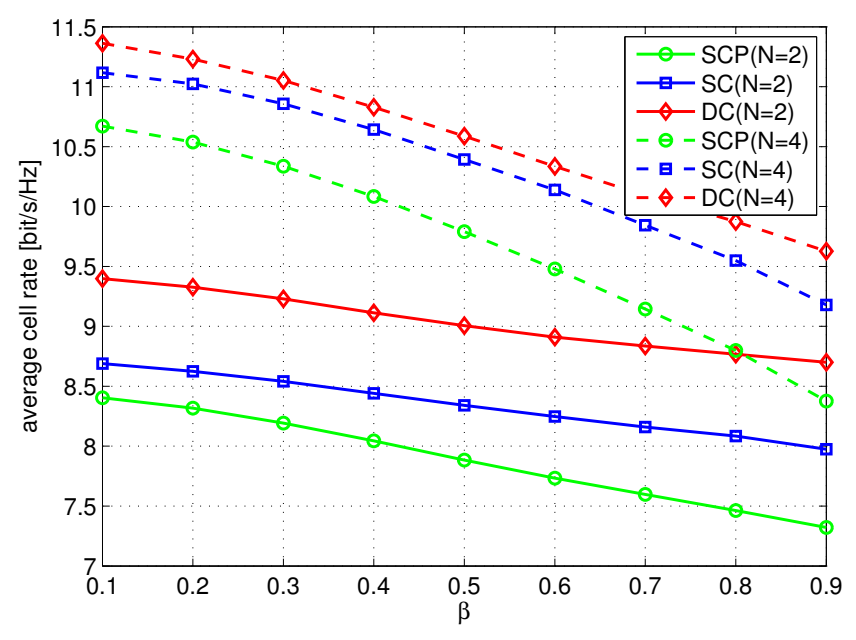

Fig. 4. Average cell rate in terms of $\beta$ when $M=4$ and $N=2,4$.

site, only partially helps the UEs close to the site border, which still suffer strong ICI. Moreover, we also observe that the performance gain achieved by DC over SCP sensibly decreases by adding more antennas at the UE. In fact, the residual ICI at the UE is higher for SCP than DC, thus IRC provides more benefits in the non-cooperative scenario. In detail, if we consider the UEs at the cell edge (Fig. 3), the performance gain achieved by DC over SCP falls down from $50 \%$ with $N=1$ to only $15 \%$ with $N=4$.

In Fig. 4 we introduce correlation among UE antennas by assuming that $\boldsymbol{R}_{\mathrm{UE}}$ is a symmetric Toeplitz matrix whose first column is $\left[\boldsymbol{R}_{\mathrm{UE}}\right]_{, 0}=\left[1, \beta, \ldots, \beta^{N-1}\right]^{T}$, and plot the average cell rate in terms of $\beta$. As expected, a higher cell rate is achieved with low-correlated antennas, i.e., for lower values of $\beta$. Then, we observe that for each of the three schemes, similar performance is achieved with few low-correlated antennas ( $N=2$ and $\beta=0.1$ ) and many high-correlated antennas ( $N=4$ and $\beta=0.9$ ). In fact, if we focus on the gain provided by IRC, it is not worthy to add more antennas at the UE when they are strongly correlated.

\section{CONCLUSiOnS}

In this paper we have extended the DC method proposed in [10] for downlink CoMP systems by assuming that UEs are equipped with multiple antennas and employ IRC. Beyond the rate improvement achieved by equipping UEs with more antennas to tackle ICI, the main finding of our work is that the gain achieved by DC over SC and SCP sensibly decreases by equipping each UE with more antennas. As clustering optimization is one of the most challenging problem in downlink CoMP systems, these results show that, when UEs can employ multiple antennas, a SC solution is a good trade off between performance and complexity.

\section{ACKNOWLEDGMent}

Part of this work has been performed in the framework of the FP7 project ICT-317669 METIS, which is partly funded by the European Union. The authors would like to acknowledge the contributions of their colleagues in METIS, although the views expressed are those of the authors and do not necessarily represent the project.

\section{REFERENCES}

[1] M. K. Karakayali, G. J. Foschini, and R. A. Valenzuela, "Network coordination for spectrally efficient communications in cellular systems," IEEE Wireless Commun. Mag., vol. 13, no. 4, pp. 56-61, Aug. 2006.

[2] D. Gesbert, S. Hanly, H. Huang, S. Shamai, O. Simeone, and W. Yu, "Multi-cell MIMO cooperative networks: a new look at interference," IEEE J. Sel. Areas Commun., vol. 28, no. 9, pp. 1380-1408, Dec. 2010.

[3] A. Papadogiannis, D. Gesbert, and E. Hardouin, "A dynamic clustering approach in wireless networks with multi-cell cooperative processing," in Proc. IEEE International Conference on Communications (ICC), Beijing (China), May 2008.

[4] J. Zhang, R. Chen, J. G. Andrews, A. Ghosh, and R. W. Heath, "Networked MIMO with clustered linear precoding," IEEE Trans. Wireless Commun., vol. 8, no. 4, pp. 1910-1921, Apr. 2009.

[5] F. Boccardi, H. Huang, and A. Alexiou, "Network MIMO with reduced backhaul requirements by MAC coordination," in Proc. IEEE Conference on Signals, Systems and Computers (Asilomar), Pacific Grove (CA), Oct. 2008.

[6] A. Papadogiannis, H. J. Bang, D. Gesbert, and E. Hardouin, "Downlink overhead reduction for multi-cell cooperative processing enabled wireless networks," in Proc. IEEE International Symposium on Personal, Indoor and Mobile Radio Communications (PIMRC), Cannes (France), Sep. 2008.

[7] J. Gong, S. Zhou, Z. Niu, L. Geng, and M. Zheng, "Joint scheduling and dynamic clustering in downlink cellular networks," in Proc. IEEE Global Communications Conference (GLOBECOM), Houston (TX), Dec. 2011.

[8] F. Boccardi, B. Clerckx, A. Ghosh, E. Hardouin, G. Jöngren, K. Kusume, E. Onggosanusi, and Y. Tang, "Multiple-antenna techniques in LTEadvanced," IEEE Commun. Mag., vol. 50, no. 3, pp. 114-121, Mar. 2012.

[9] I. Hwang, C.-B. Chae, J. Lee, and R. W. Heath, "Multicell cooperative systems with multiple receive antennas," IEEE Wireless Commun. Mag., vol. 20, no. 1, pp. 50-58, Feb. 2013.

[10] P. Baracca, F. Boccardi, and V. Braun, "A dynamic joint clustering scheduling algorithm for downlink CoMP systems with limited CSI," in Proc. IEEE International Symposium on Wireless Communication Systems (ISWCS), Paris (France), Aug. 2012.

[11] J. Winters, "Optimum combining in digital mobile radio with cochannel interference," IEEE J. Sel. Areas Commun., vol. 2, no. 4, pp. 528-539, Jul. 1984.

[12] J. P. Kermoal, L. Schumacher, K. I. Pedersen, P. E. Mogensen, and F. Frederiksen, "A stochastic MIMO radio channel model with experimental validation," IEEE J. Sel. Areas Commun., vol. 20, no. 6, pp. 1211-1226, Aug. 2002.

[13] S. Sesia, I. Toufik, and M. Baker, LTE: The UMTS Long Term Evolution. John Wiley \& Sons, 2009. 